\title{
Analytic bounds and approximations for annuities and Asian options.
}

\author{
Steven Vanduffel* \\ Katholieke Universiteit Leuven \\ Zhaoning Shang \\ Luc Henrard \\ Katholieke Universiteit Leuven \\ Fortis \\ Jan Dhaene \\ Katholieke Universiteit Leuven \\ Emiliano A. Valdez \\ University of New South Wales
}

\begin{abstract}
Even in case of the Brownian motion as most natural rate of return model it appears too difficult to obtain analytic expressions for most risk measures of constant continuous annuities. In literature so-called comonotonic approximations have been proposed but these still require the evaluation of integrals. In this paper we show that these integrals can sometimes be computed, and we obtain explicit approximations for some popular risk measures for annuities.

Next, we show how these results can be used to obtain fully analytic expressions for lower and upper bounds for the price of a continuously sampled European-style Asian option with fixed exercise price. These analytic lower bound prices are as sharp as those from Rogers \& Shi (1995), if not sharper, but in contrast do not require any longer the evalution of a two-dimensional or a onedimensional integral.
\end{abstract}

Keywords: Asian option, Brownian motion, analytic, closed-form, lower bound, annuity, fixed strike, continuously sampled.

\footnotetext{
${ }^{*}$ Corresponding author : Steven Vanduffel, Assistant Professor, Katholieke Universiteit Leuven, Naamsestraat 69, 3000 Leuven, Belgium (e-mail: Steven.Vanduffel@econ.kuleuven.be).
} 


\section{Introduction}

Many risk management problems in finance and insurance involve a random variable (rv) $S$ given by

$$
S=\sum_{i=1}^{n} \alpha_{i} e^{Z_{i}}
$$

where the $\alpha_{i}$ are non-negative real numbers and $\left(Z_{1}, Z_{2}, \ldots, Z_{n}\right)$ is a random vector of either returns or discount factors. The means of the $Z_{i}$ will be denoted by $E\left[Z_{i}\right]$ and their variances by $\sigma_{Z_{i}}^{2}$. Applications such as the valuation of Asian or basket options, the setting of provisions and optimal portfolio selection problems, amongst others, come down to the evaluation of risk measures related to the cumulative distribution function (cdf) of a random variable $S$ as defined in (1).

We define a risk measure as a mapping from the set of random variables, usually representing the risks at hand, to the set of real numbers $\mathbb{R}$. Risk measures are a helpful tool for decision making because they summarise the information available about the random variable $X$ into one single number $\rho[X]$.

In this paper we consider the $p$-quantile risk measure, denoted by $Q_{p}[X]$, and which for any $p$ in $(0,1)$ is defined as

$$
Q_{p}[X]=\inf \left\{x \in \mathbb{R} \mid F_{X}(x) \geq p\right\}, \quad p \in(0,1),
$$

where $F_{X}(x)=\operatorname{Pr}[X \leq x]$. We will also consider the Conditional Tail Expectation, denoted by $\mathrm{CTE}_{p}[X]$, and defined as

$$
C T E_{p}[X]=E\left[X \mid X>Q_{p}[X]\right], \quad p \in(0,1) .
$$

In this paper we will only deal with random variables with a continuous and strictly increasing cdf. Then it follows that

$$
C T E_{p}[X]=\frac{\int_{p}^{1} Q_{q}[X] d q}{1-p}, \quad p \in(0,1),
$$

see e.g. Dhaene et al. (2006). Using the notation $(x-d)_{+}=\max (x-d, 0)$ we will also define the stop-loss premium with retention $d$ of the random variable $X$ as $E\left[(X-d)_{+}\right]$. It can be easily shown that

$$
E\left[(X-d)_{+}\right]=\int_{d}^{\infty}\left(1-F_{X}(x)\right) d x, \quad-\infty<d<+\infty .
$$

and also that

$$
E\left[(X-d)_{+}\right]=(1-p)\left(C T E_{p}[X]-d\right),
$$

where $d=Q_{p}[X]$.

In this paper we will also compare different rv's using the concept of convex orderings. A $\mathrm{rv} X$ is convex smaller than the $\mathrm{rv} Y$, denoted by $X \leq_{c x} Y$, if $E[X]=$ $E[Y]$ and $E\left[(X-d)_{+}\right] \leq E\left[(Y-d)_{+}\right]$for $-\infty<d<+\infty$. The convex ordering reflects the common preferences of all risk averse decision makers when choosing between rv's with equal mean. This holds in both the classical utility theory from von Neuman \& 
Morgenstern as in Yaari's dual utility theory; see e.g. Denuit et al. (1999) or Dhaene et al. (2006) for more details.

The discrete case (sums of random variables) has a continuous counterpart (integrals of stochastic processes), and in this paper we mainly focus on developing some explicit results when the stochastic process under consideration is a geometric Brownian motion which is the continuous equivalent of the discrete Gaussian setting. Consequently, in line with Dhaene et al. (2002) we will consider in this paper the continuous equivalent of (1), which is the continuous temporary annuity $S_{t}$ defined by

$$
S_{t}=\int_{0}^{t} \alpha(\tau) e^{Z(\tau)} d \tau
$$

where $\{Z(\tau), \tau \geq 0\}$ represents a Brownian motion, i.e. the process has independent and stationary increments, $Z(0)=0$, and for any $\tau \geq 0$ the random variable $Z(\tau)$ is normally distributed with mean $-\delta \tau$ and variance $\sigma^{2} \tau$. As some of our findings will also hold for negative discount rates and are useful to derive results for the compounding case, we will not make a general assumption on the sign of $\delta$. Finally, the continuous stream of payments is described by a non-negative function of $\alpha(\tau)$. In the remainder of the paper we tacitly assume all integrals to be well defined.

The random variable $S_{t}$ can be interpreted as the stochastically discounted value of all future obligations $\alpha(\tau)$. Investing an initial amount equal to $Q_{p}\left[S_{t}\right]$ according to this return process enables one to meet all future payments with probability $p$.

Although some analytic results are known for the annuity $S_{t}$ 's distribution function, no "closed-form" expression appears to exist; see e.g. De Schepper et al. (1994, 1995). It was already mentioned in Dhaene et al. (2002) that using the so-called comonotonic upper and lower bound approximations for risk measures of constant continuous annuities gives rise to expressions that only involve the evaluation of a single integral. However, Huang et al. (2004) mentioned that numerical evaluations of these integrals are sensitive to the granularity that is used to approximate the integral by a sum.

In this paper we show that it is sometimes possible to evaluate these integrals explicitly. First, we show that in case of a constant continuous annuity (7) the comonotonic upper bound approximation gives rise to closed-form results for the quantiles, conditional tail expectation and stop-loss premiums. Next, we demonstrate that for some specific choices of the conditioning random variable $\Lambda$ explicit results for these risk measures can also be obtained when using the comonotonic lower bound approximations, and we will compare these with available explicit results for perpetuities. Finally, we use these results to obtain fully analytic expressions for a lower and a upper bound for a continuously sampled European-style Asian option with fixed strike. The lower bound prices are as sharp as those from Rogers \& Shi (1995) but in contrast do not require any longer the evaluation of integrals.

The remainder of this paper has been organised as follows. In Section 2, as an analogue of the continuous case developed in this paper, we recall from the literature the general results regarding the comonotonic bounds for the discrete case of the sum in (1). In Section 3 we develop explicit expressions for upper and lower bounds of several risk measures for a constant continuous annuity, and in Section 4 we will compare these approximations with available explicit results in the case of constant continuous perpetuities. In Sections 5 we apply our results to the analysis of Asian options. Finally, Section 6 provides some concluding remarks. 


\section{Comonotonic approximations - the discrete case}

\section{$2.1 \quad$ General results}

Let the random variable $S$ be given by (1), where the $\alpha_{i}$ are non-negative real numbers and the random vector $\left(Z_{1}, Z_{2}, \ldots, Z_{n}\right)$ has a multivariate Normal distribution. Consider the conditioning random variable $\Lambda$ which is given by

$$
\Lambda=\sum_{i=1}^{n} \gamma_{i} Z_{i}
$$

for some $\gamma_{i}$. Following Kaas et al. (2000) we define a random variable $S^{l}$ as

$$
S^{l} \stackrel{\text { def }}{=} E[S \mid \Lambda]=\sum_{i=1}^{n} \alpha_{i} e^{E\left[Z_{i}\right]+\frac{1}{2}\left(1-r_{i}^{2}\right) \sigma_{Z_{i}}^{2}+r_{i} \sigma_{Z_{i}} \frac{\Lambda-E[\Lambda]}{\sigma_{\Lambda}}}
$$

where $r_{i}$ denotes the correlation coefficient between $Z_{i}$ and $\Lambda$, for $i=1, \ldots, n$. We find for the cdf of $S^{l}$ that

$$
S^{l} \stackrel{\mathrm{d}}{=} \sum_{i=1}^{n} \alpha_{i} e^{E\left[Z_{i}\right]+\frac{1}{2}\left(1-r_{i}^{2}\right) \sigma_{Z_{i}}^{2}+r_{i} \sigma_{Z_{i}} \Phi^{-1}(U)},
$$

with $\Phi$ the cdf of a standard normally distributed rv and $U$ a uniformly distributed rv on the unit interval.

Then, provided all coefficients $r_{i}$ are positive we find that the components in the sum are all increasing in $\Lambda$, meaning that they are co-monotonic, so that we can invoke a well-known additivity property for risk measures of sums of comonotonic risks to find that for $p \in(0,1)$,

$$
\begin{aligned}
Q_{p}\left[S^{l}\right] & =\sum_{i=1}^{n} \alpha_{i} e^{E\left[Z_{i}\right]+\frac{1}{2}\left(1-r_{i}^{2}\right) \sigma_{Z_{i}}^{2}+r_{i} \sigma_{Z_{i}} \Phi^{-1}(p)}, \\
C T E_{p}\left[S^{l}\right] & =\sum_{i=1}^{n} \alpha_{i} e^{E\left[Z_{i}\right]+\frac{1}{2} \sigma_{Z_{i}}^{2}} \frac{\Phi\left(r_{i} \sigma_{Z_{i}}-\Phi^{-1}(p)\right)}{1-p}
\end{aligned}
$$

We refer to Kaas et al. (2000) or Dhaene et al. (2002) for more details.

Finally, from (6) we find that:

$$
E\left[\left(S^{l}-d\right)_{+}\right]=\sum_{i=1}^{n} \alpha_{i} e^{E\left[Z_{i}\right]+\frac{1}{2} \sigma_{Z_{i}}^{2}} \Phi\left(r_{i} \sigma_{Z_{i}}-\Phi^{-1}(p)\right)-d(1-p),
$$

where $Q_{p}\left[S^{l}\right]=d$. In literature also another rv, denoted by $S^{c}$, has been proposed to approximate the risk measures of $S$; see e.g. Kaas et al. (2000) or Dhaene et al. (2002). In our lognormal context $S^{c}$ can be defined by imposing the correlations in expression (10) to be equal to one. Formally we obtain

$$
S^{c} \stackrel{d}{=} \sum_{i=1}^{n} \alpha_{i} e^{\mathrm{E}\left[Z_{i}\right]+\sigma_{Z_{i}} \Phi^{-1}(U)},
$$

and we obtain closed from expressions for $Q_{p}\left[S^{c}\right], C T E_{p}\left[S^{c}\right]$ and $E\left[\left(S^{c}-d\right)_{+}\right]$by applying the substitution $r_{i}=1$ in the equivalent expressions (11), (12) and (13) for the rv $S^{l}$. 
As demonstrated in Kaas et al. (2000), it follows that the rv's $S, S^{l}$ and $S^{c}$ are convex ordered:

$$
S^{l} \leq_{c x} S \leq_{c x} S^{c}
$$

and this will imply the following ordering relations:

$$
\operatorname{Var}\left[S^{l}\right] \leq \operatorname{Var}[S] \leq \operatorname{Var}\left[S^{c}\right], \quad p \in(0,1),
$$

and

$$
\begin{aligned}
& E\left[\left(S^{l}-d\right)_{+}\right] \leq E\left[(S-d)_{+}\right] \leq E\left[\left(S^{c}-d\right)_{+}\right], \quad d \in \mathbb{R} . \\
& C T E_{p}\left[S^{l}\right] \leq C T E_{p}[S] \leq C T E_{p}\left[S^{c}\right], \quad p \in(0,1),
\end{aligned}
$$

All the above mentioned results can also be found in Dhaene et al. (2002).

\subsection{The optimal choice for the conditioning random variable}

The best closed-form approximations based on the lower bound $E[S \mid \Lambda]$ will occur when $\Lambda$ is chosen such that $\mathrm{E}[S \mid \Lambda]$ is sufficiently 'close' to the rv $S$ while still finding an explicit expression for it. Note that when we take $\Lambda \equiv S$ we find that $\mathrm{E}[S \mid \Lambda] \equiv S$ but this ideal choice for $\Lambda$ is not feasible because it does not allow explicit calculations.

From (16) and (17) we find that a good conditioning $\mathrm{rv} \Lambda$ could be defined as one that allows explicit calculations for $\mathrm{E}[S \mid \Lambda]$ while obtaining as large as possible values for $\operatorname{Var}[\mathrm{E}[S \mid \Lambda]]$ or, alternatively, for $\mathrm{CTE}_{p}[\mathrm{E}[S \mid \Lambda]]$. Note that the latter approach is more focusing on finding optimal approximations for $S$ in case $S$ takes large values whereas the former intends to provide a global optimal fit to $S$.

Vanduffel et al. (2005a) proved that the first order approximation of the variance of $S^{l}$ will be maximised when $\Lambda$ is given by

$$
\Lambda=\sum_{i=1}^{n} \alpha_{i} e^{E\left[Z_{i}\right]+\frac{1}{2} \sigma_{Z_{i}}^{2}} Z_{i} .
$$

This choice for $\Lambda$ is denoted by $\Lambda^{M V}$. When the approximations for the risk measures of $S^{l}$ are based on $\Lambda^{M V}$ as conditioning random variable we call these in the paper 'maximal variance' approximations.

It was shown in Vanduffel et al. (2007) that the following choice for $\Lambda$ maximises a first-order approximation of $C T E_{p}\left[S^{l}\right]$ :

$$
\Lambda=\sum_{i=1}^{n} \alpha_{i} e^{E\left[Z_{i}\right]+\frac{1}{2} \sigma_{Z_{i}}^{2}} \cdot \Phi^{\prime}\left(r_{i}^{M V} \sigma_{Z_{i}}-\Phi^{-1}(p)\right) Z_{i} .
$$

where $r_{j}^{M V}$ is the correlation between $\Lambda^{M V}$ and $Z_{i}$. This choice for $\Lambda$ will be denoted by $\Lambda^{(p)}$, and note that $\Lambda^{(p)}$ depends on the probability level $p$ reflecting that it is indeed constructed to be locally optimal in some sense. When $\Lambda^{(p)}$ is used to derive the lower bound approximations we will call these 'maximal CTE' approximations. For a detailed account on the topic of appropriately choosing the conditioning $\mathrm{rv} \Lambda$ we refer to Vanduffel et al. (2007). 


\section{Closed-form comonotonic approximations for the con- tinuous case}

\subsection{General results}

We will now focus on the temporary continuous annuity $S_{t}$ as defined in (7). Analogous to the discrete setting discussed in Kaas et al. (2000) it was stated in Dhaene et al. (2002) that $S_{t}^{l} \leq_{c x} S_{t} \leq_{c x} S_{t}^{c}$, where the random variable $S_{t}^{c}$ is now defined by

$$
S_{t}^{c} \stackrel{d}{=} \int_{0}^{t} \alpha(\tau) e^{-\delta \tau+\sigma \sqrt{\tau} \Phi^{-1}(U)} d \tau,
$$

with $U$ a uniformly $(0,1)$ distributed random variable. For $S_{t}^{l}$ we have that

$$
S_{t}^{l} \stackrel{\text { def }}{=} E\left[S_{t} \mid \Lambda\right]=\int_{0}^{t} \alpha(\tau) e^{-\delta \tau+\frac{1}{2} \sigma^{2} \tau\left(1-r^{2}(\tau)\right)+r(\tau) \sigma \sqrt{\tau} \frac{\Lambda-E[\Lambda]}{\sigma_{\Lambda}}} d \tau,
$$

where $r(\tau)$ is the correlation between $Z(\tau)$ and the conditioning $\operatorname{rv} \Lambda$ which is defined as

$$
\Lambda=\int_{0}^{t} \gamma(\tau) Z(\tau) d \tau
$$

for some function $\tau \rightarrow \gamma(\tau)$. The cdf of $S_{t}^{l}$ is given by:

$$
S_{t}^{l} \stackrel{d}{=} \int_{0}^{t} \alpha(\tau) e^{-\delta \tau+\frac{1}{2} \sigma^{2} \tau\left(1-r^{2}(\tau)\right)+r(\tau) \sigma \sqrt{\tau} \Phi^{-1}(V)} d \tau
$$

with $V$ a rv that is uniformly distributed on the unit interval.

Note that $S_{t}^{c}$ will be an integral of comonotonic random variables. As a result, its quantiles and conditional tail expectations follow from

$$
\begin{aligned}
Q_{p}\left[S_{t}^{c}\right] & =\int_{0}^{t} \alpha(\tau) e^{-\delta \tau+\sigma \sqrt{\tau} \Phi^{-1}(p)} d \tau, \\
C T E_{p}\left[S_{t}^{c}\right] & =\int_{0}^{t} \alpha(\tau) e^{-\delta \tau+\sigma^{2} \tau / 2} \frac{\Phi\left(\sigma \sqrt{\tau}-\Phi^{-1}(p)\right)}{1-p} d \tau,
\end{aligned}
$$

with $0<p<1$. The stop-loss premiums can be easily calculated from this using equation (6).

When the function $f(\tau)=\operatorname{Cov}[Z(\tau), \Lambda]$ is non-negative we also find that $S_{t}^{l}$ will be an integral of comonotonic random variables and we obtain

$$
\begin{aligned}
Q_{p}\left[S_{t}^{l}\right] & =\int_{0}^{t} \alpha(\tau) e^{-\delta \tau+\frac{1}{2} \sigma^{2} \tau\left(1-r^{2}(\tau)\right)+r(\tau) \sigma \sqrt{\tau} \Phi^{-1}(p)} d \tau, \\
C T E_{p}\left[S_{t}^{l}\right] & =\int_{0}^{t} \alpha(\tau) e^{-\delta \tau+\sigma^{2} \tau / 2} \frac{\Phi\left(r(\tau) \sigma \sqrt{\tau}-\Phi^{-1}(p)\right)}{1-p} d \tau,
\end{aligned}
$$

with again $0<p<1$. Also in this case we use (6) to derive the lower bound expressions for the stop-loss premiums. The results (24)-(27) can also be found in Dhaene et al. (2002).

We note that the formulas (24)-(27) involve the evaluation of an integral and as such may require a numerical evaluation. Dhaene et al. (2002) as well as Huang et al. (2004) effectively substitute $\alpha(\tau)$ by $\sum_{i=1}^{n} \alpha\left(\frac{i t}{n}\right) \Delta_{\tau=\frac{i t}{n}}$ where the symbol $\Delta$ is here 
used to denote Dirac's delta function. This will effectively turn the integral at hand into a discrete sum and it follows that we recover the discrete results as mentioned in Section 2; See also Lord (2006).

However, in case of constant continuous annuities it is sometimes possible to directly evaluate the integrals and this will be discussed in the next subsections. In what follows the results will be valid for $\alpha(\tau)$ being a constant, but for simplicity of the exposition we will consider a continuous stream of unit cash flows, i.e. $\alpha(\tau)=1$. In the remainder of the paper we will also make use of the quantity $\delta^{*}$ which is defined as:

$$
\delta^{*}=\delta-\frac{1}{2} \sigma^{2}
$$

\subsection{Upper bound approach}

In this section we will derive closed-form approximations for several of the risk measures of $S_{t}^{c}$. These explicit formulas will only hold when $\delta>0$.

The quantiles of $S_{t}^{c}$ follow from (24) but now with $\alpha(\tau)=1$. Then, by substituting $y=\sqrt{\tau}$ and realising that the resulting integral can be rewritten in terms of the standard-normal cdf $\Phi$ we find the following analytical expression for $Q_{p}\left[S_{t}^{c}\right]$ :

$$
Q_{p}\left[S_{t}^{c}\right]=\frac{1}{\delta}-\frac{1}{\delta} e^{-\delta t+\sigma \sqrt{t} \Phi^{-1}(p)}+\frac{1}{\delta} \sqrt{2 \pi} a e^{\frac{a^{2}}{2}}(\Phi(\sqrt{2 \delta t}-a)-\Phi(-a)),
$$

with

$$
a=\frac{\sigma \Phi^{-1}(p)}{\sqrt{2 \delta}}, \quad \delta>0, \quad(0<p<1) .
$$

A detailed proof for this result can be found in Appendix A.

Furthermore, from (25) and using the same substitution $y=\sqrt{\tau}$ as in the case of the quantiles, we find after some detailed computations the following closed-form expression for the conditional tail expectations $C T E_{p}\left[S_{t}^{c}\right]$ :

$$
\begin{aligned}
C T E_{p}\left[S_{t}^{c}\right]= & \frac{1}{\delta^{*}}-\frac{1}{\delta^{*}} e^{-\delta^{*} t} \frac{\Phi\left(\sigma \sqrt{t}-\Phi^{-1}(p)\right)}{1-p} \\
& +\frac{\sigma}{\delta^{*}} e^{\frac{a^{2}-\left(\Phi^{-1}(p)\right)^{2}}{2}} \sqrt{\frac{1}{2 \delta}} \frac{\Phi(\sqrt{2 \delta t}-a)-\Phi(-a)}{1-p}, \quad(0<p<1) .
\end{aligned}
$$

Finally, the stop-loss premiums $E\left[\left(S^{c}-d\right)_{+}\right]$with retentions $d>0$ can be derived from (6), (31) and (29).

\subsection{Lower bound approaches}

\subsubsection{General results}

In order to compute the risk measures of $S_{t}^{l}$ we may suggest to use the continuous analogon of (18) namely the conditioning random variable $\Lambda=\int_{0}^{t} e^{-\delta^{*} \tau} Z(\tau) d \tau$. This choice will ensure that $\Lambda$ is 'close' to $S_{t}$ because one easily can prove along similar lines as in Vanduffel et al. (2005a) it will maximise the first order Taylor approximation 
for the variance of $S_{t}^{l}$. In this case we find that $\Lambda$ is normally distributed with mean 0 and variance given by

$$
\begin{aligned}
\sigma_{\Lambda}^{2}=\operatorname{Var}[\Lambda] & =\int_{0}^{t} \int_{0}^{t} e^{-\delta^{*}(\tau+\nu)} \min (\tau, \nu) d \tau d \nu \\
& =\frac{1}{2 \delta^{* 3}}+\frac{3+2 \delta^{*} t-4 e^{\delta^{*} t}}{2 \delta^{* 3} e^{2 \delta^{*} t}}
\end{aligned}
$$

and $r(\tau)$ is given by

$$
r(\tau)=\frac{\operatorname{Cov}[Z(\tau), \Lambda]}{\sigma_{\Lambda} \sigma \sqrt{\tau}}=\frac{1}{\sigma_{\Lambda} \sqrt{\tau}}\left[\frac{1-e^{-\delta^{*} \tau}}{\delta^{* 2}}-\frac{\tau e^{-\delta^{*} t}}{\delta^{*}}\right], \quad \tau \leq t .
$$

Since the function $f(\tau)=\operatorname{Cov}[Z(\tau), \Lambda]$ is a non-negative function, $S_{t}^{l}$ will be an integral of comonotonic random variables. Unfortunately, for finite annuities, it appears that there are no closed-form solutions for the quantiles, conditional tail expectations and stop-loss premiums of $S_{t}^{l}$ when using this choice for $\Lambda$. We now propose other choices for $\Lambda$ that will allow to obtain explicit closed-form approximations for these risk measures.

\subsection{2 $\Lambda=\int_{0}^{\infty} e^{-\delta^{*} \tau} Z(\tau) d \tau$}

Note that this choice for $\Lambda$ implicitly assumes that $\delta^{*}>0$ and the formulas we derive are only valid in this case. Then, the quantiles of $S_{t}^{l}(0<p<1)$ follow from (26) with $\alpha(\tau)=1$. Then, by substituting $y=\sqrt{\tau}$ and realising that

$$
r(\tau)=\sqrt{\frac{2}{\delta^{*} \tau}}\left(1-e^{-\delta^{*} \tau}\right) .
$$

we find that they can be expressed as

$$
Q_{p}\left[S_{t}^{l}\right]=\int_{0}^{t} e^{-\delta^{*} \tau-\frac{1}{2} c^{2}\left(1-e^{-\delta^{*} \tau}\right)^{2}+c \Phi^{-1}(p)\left(1-e^{-\delta^{*} \tau}\right)} d \tau,
$$

with

$$
c=\sigma \sqrt{\frac{2}{\delta^{*}}} .
$$

By making the substitution $y=c\left(1-e^{-\delta^{*} \tau}\right)$ one sees that the integral (35) can be rewritten in terms of the standard-normal cdf. As a result we find the following analytical expression for the quantiles of $S_{t}^{l}$ :

$$
Q_{p}\left[S_{t}^{l}\right]=\frac{1}{c \delta^{*}} \sqrt{2 \pi} e^{\frac{\left(\Phi^{-1}(p)\right)^{2}}{2}}\left(\Phi\left(k_{t}\right)-1+p\right),
$$

with

$$
k_{t}=c\left(1-e^{-\delta^{*} t}\right)-\Phi^{-1}(p)
$$

After some tedious algebra we also find that

$$
\begin{aligned}
C T E_{p}\left[S_{t}^{l}\right]= & \frac{1}{\delta^{*}(1-p)} \Phi\left(k_{t}\right)\left(1-e^{-\delta^{*} t}\right) \\
& +\frac{1}{\delta^{*}(1-p)}\left(\frac{\Phi^{-1}(p)}{c}\right)\left(1-p-\Phi\left(k_{t}\right)\right) \\
& -\frac{1}{\sqrt{2 \pi} c \delta^{*}(1-p)}\left(e^{-\frac{1}{2}\left(\Phi^{-1}(p)\right)^{2}}-e^{-\frac{1}{2} k_{t}^{2}}\right),
\end{aligned}
$$


Finally, invoking (6) again we find from the above expressions also the stop-loss premiums of $S_{t}^{l}$ with retentions $d>0$.

We point out that this specific choice for $\Lambda=\int_{0}^{\infty} e^{-\delta^{*} \tau} Z(\tau) d \tau$ will not always perform very well for finite annuities. However, when $t$ reaches infinity, this choice for $\Lambda$ leads to the continuous equivalent of the 'maximal variance' lower bound approach that we discussed in Section 2. Consequently our specific choice for $\Lambda$ is likely to yield excellent results when $t$ reaches infinity whilst allowing an analytical expression for the selected risk measures of $S_{t}^{l}$ for finite $t$ as well.

\subsection{3 $\Lambda=\sum_{i=1}^{n} \gamma\left(t_{i}\right) Z\left(t_{i}\right)$}

The approximations we derive in this subsection will exist for all values for $\delta$. We will assume that $0<t_{1}<t_{2}<\ldots<t_{n}=t$ and $\gamma\left(t_{i}\right)>0$ for $i=1,2, \ldots, n-1$ whereas $\gamma\left(t_{n}\right) \geq 0$. We will also take $t_{0}=0$ and introduce the notation $S_{t}^{l, s}$ to denote the convex lower bound for $S_{t}$. From (26) we see that the quantiles of $S_{t}^{l, s}(0<p<1)$ can also be expressed as

$$
Q_{p}\left[S_{t}^{l, s}\right]=\int_{0}^{t} e^{-\delta^{*} \tau-\frac{\operatorname{Cov}^{2}[\Lambda, Z(\tau)]}{2 \sigma_{\Lambda}^{2}}+\frac{\operatorname{Cov}[\Lambda, Z(\tau)]}{\sigma_{\Lambda}} \Phi^{-1}(p)} d \tau .
$$

Here, for $0 \leq \tau<t$ we have that $\operatorname{Cov}[\Lambda, Z(\tau)]$ can be expressed as

$$
\operatorname{Cov}[\Lambda, Z(\tau)]=\sum_{j=0}^{n-1}\left(a_{j}+b_{j} \tau\right) 1_{t_{j \leq \tau<t_{j+1}}}
$$

where $1_{()}$is the usual notation for the indicator function and

$$
\begin{aligned}
& a_{j}=\sigma^{2} \sum_{i=1}^{j} \gamma\left(t_{i}\right) t_{i}, \\
& b_{j}=\sigma^{2} \sum_{i=j+1}^{n} \gamma\left(t_{i}\right), \quad j=0,1, \ldots, n-1,
\end{aligned}
$$

where we conventially assumed that $\sum_{i=1}^{0} x_{i}=0$. Moreover, $\sigma_{\Lambda}^{2}$ is given by

$$
\sigma_{\Lambda}^{2}=\sigma^{2} \sum_{i=1}^{n} \sum_{j=1}^{n} \gamma\left(t_{i}\right) \gamma\left(t_{j}\right) \min \left(t_{i}, t_{j}\right)
$$

Then, we find that

$$
Q_{p}\left[S_{t}^{l, s}\right]=\sum_{j=0}^{n-2} c_{j} e^{\frac{d_{j}^{2}}{2 e_{j}}} \sqrt{\frac{2 \pi}{e_{j}}}\left(\Phi\left(t_{j+1} \sqrt{e_{j}}-\frac{d_{j}}{\sqrt{e_{j}}}\right)-\Phi\left(t_{j} \sqrt{e_{j}}-\frac{d_{j}}{\sqrt{e_{j}}}\right)\right)+f_{n-1},
$$

where in case $\gamma\left(t_{n}\right) \neq 0$ we have that $f_{n-1}$ is given by

$$
f_{n-1}=c_{n-1} e^{\frac{d_{n-1}^{2}}{2 e_{n-1}}} \sqrt{\frac{2 \pi}{e_{n-1}}}\left(\Phi\left(t_{n} \sqrt{e_{n-1}}-\frac{d_{n-1}}{\sqrt{e_{n-1}}}\right)-\Phi\left(t_{n-1} \sqrt{e_{n-1}}-\frac{d_{n-1}}{\sqrt{e_{n-1}}}\right)\right),
$$


while when $\gamma\left(t_{n}\right)=0$ we find that

$$
f_{n-1}=\frac{c_{n-1}}{\delta^{*}}\left(e^{-\delta^{*} t_{n-1}}-e^{-\delta^{*} t_{n}}\right) .
$$

Here, for $j=0,1, \ldots, n-1$ we have that

$$
\begin{aligned}
c_{j} & =e^{-\frac{a_{j}^{2}}{2 \sigma_{\Lambda}^{2}}+\frac{a_{j}}{\sigma_{\Lambda}} \Phi^{-1}(p)}, \\
d_{j} & =-\delta^{*}-\frac{a_{j} b_{j}}{\sigma_{\Lambda}^{2}}+\frac{b_{j}}{\sigma_{\Lambda}} \Phi^{-1}(p), \\
e_{j} & =\frac{1}{\sigma_{\Lambda}^{2}} b_{j}^{2},
\end{aligned}
$$

For the conditional tail expectations we find after some lengthy computations that

$$
\begin{aligned}
C T E_{p}\left[S_{t}^{l, s}\right]= & \frac{1}{1-p} \sum_{j=0}^{n-2}\left(\frac{e^{-\delta^{*} t_{j}}}{\delta^{*}} \Phi\left(\kappa_{j} t_{j}+\lambda_{j}\right)-\frac{e^{-\delta^{*} t_{j+1}}}{\delta^{*}} \Phi\left(\kappa_{j} t_{j+1}+\lambda_{j}\right)\right. \\
& +\frac{1}{\delta^{*}} e^{\frac{1}{2}\left(\frac{\delta^{*}}{\kappa_{j}}\right)^{2}+\frac{\lambda_{j}}{\kappa_{j}} \delta^{*}} \times\left(\Phi\left(\kappa_{j} t_{j+1}+\lambda_{j}+\frac{\delta^{*}}{\kappa_{j}}\right)\right. \\
& \left.-\Phi\left(\kappa_{j} t_{j}+\lambda_{j}+\frac{\delta^{*}}{\kappa_{j}}\right)\right)+\nu_{n-1},
\end{aligned}
$$

where in case $\gamma\left(t_{n}\right) \neq 0$ we have that $\nu_{n-1}$ is given by

$$
\begin{aligned}
\nu_{n-1}= & \frac{1}{(1-p) \delta^{*}}\left(e^{-\delta^{*} t_{n-1}} \Phi\left(\kappa_{n-1} t_{n-1}+\lambda_{n-1}\right)-e^{-\delta^{*} t_{n}} \Phi\left(\kappa_{n-1} t_{n}+\lambda_{n-1}\right)\right. \\
& +e^{\frac{1}{2}\left(\frac{\delta^{*}}{\kappa_{n-1}}\right)^{2}+\frac{\lambda_{n-1}}{\kappa_{n-1}} \delta^{*}} \times\left(\Phi\left(\kappa_{n-1} t_{n}+\lambda_{n-1}+\frac{\delta^{*}}{\kappa_{n-1}}\right)-\Phi\left(\kappa_{n-1} t_{n-1}+\lambda_{n-1}+\frac{\delta^{*}}{\kappa_{n-1}}\right)\right),
\end{aligned}
$$

while for $\gamma\left(t_{n}\right)=0$ it proofs that $\nu_{n-1}$ is given by

$$
\nu_{n-1}=\frac{\Phi\left(\lambda_{n-1}\right)}{(1-p) \delta^{*}}\left(e^{-\delta^{*} t_{n-1}}-e^{-\delta^{*} t_{n}}\right) .
$$

Here, the coefficients $\kappa_{j}$ and $\lambda_{j}(j=0,1, \ldots, n-1)$ are given by

$$
\begin{aligned}
\kappa_{j} & =\frac{b_{j}}{\sigma_{\Lambda}}, \\
\lambda_{j} & =\frac{a_{j}}{\sigma_{\Lambda}}-\Phi^{-1}(p) .
\end{aligned}
$$

Finally, the stop-loss premiums for $S_{t}^{l, s}$ follow from (6), (48) and (44).

\section{Perpetuities}

Consider the perpetuity $S_{\infty}$ defined by

$$
S_{\infty}=\int_{0}^{\infty} e^{Z(\tau)} d \tau .
$$


Dufresne (1990) proved that its reciprocal $S_{\infty}^{-1}$ is Gamma distributed with parameters $\frac{2 \delta}{\sigma^{2}}$ and $\frac{\sigma^{2}}{2}$ meaning that its probability density function (pdf) is expressed as

$$
f_{S_{\infty}^{-1}}(x ; \alpha, \beta)=\frac{1}{\beta^{\alpha} \Gamma(\alpha)} x^{\alpha-1} e^{-x / \beta}, \quad x>0,
$$

where $\alpha>0, \beta>0$ and $\Gamma($.$) denotes the Gamma function:$

$$
\Gamma(\alpha)=\int_{0}^{\infty} u^{\alpha-1} e^{-u} d u \quad(\alpha>0) .
$$

It follows that $S_{\infty}$ has as pdf:

$$
f_{S_{\infty}}(y ; \alpha, \beta)=f_{S_{\infty}}(1 / y ; \alpha, \beta) / y^{2}, \quad y>0,
$$

with quantiles and conditional tail expectations given by

$$
Q_{p}\left[S_{\infty}\right]=\frac{1}{F_{S_{\infty}^{-1}}^{-1}(1-p ; \alpha, \beta)}, \quad p \in(0,1),
$$

and

$$
C T E_{p}\left[S_{\infty}\right]=\frac{F_{S_{\infty}^{-1}}\left(F_{S_{\infty}^{-1}}^{-1}(1-p ; \alpha, \beta) ; \alpha-1, \beta\right)}{(1-p)(\alpha-1) \beta}, \quad p \in(0,1),
$$

where $F_{S_{\infty}^{-1}}(. ; \alpha, \beta)$ is the cdf of the Gamma distribution with parameters $\alpha$ and $\beta$. Note that expression (57) implicitly assumes that $\delta>\frac{\sigma^{2}}{2}$. Since the Gamma distribution is readily available in many statistical software packages these different risk measures can be determined easily.

Hence, we can compare the cumulative distribution functions of the lower bound $S_{\infty}^{l}$ and the upper bound $S_{\infty}^{c}$ with the exact cumulative distribution function of $S_{\infty}$. For the lower bound approach we take $\Lambda=\int_{0}^{\infty} e^{-\delta^{*} \tau} Z(\tau) d \tau$ as conditioning rv.

From (56), (29) and (37), with $t \rightarrow \infty$, we find for $0<p<1$ the following closed-form expressions for the quantiles of $S_{\infty}, S_{\infty}^{c}$ and $S_{\infty}^{l}$, respectively.

$$
\begin{aligned}
Q_{p}\left[S_{\infty}\right] & =\frac{1}{F_{S_{\infty}^{-1}}^{-1}\left(1-p ; \frac{2 \delta}{\sigma^{2}}, \frac{\sigma^{2}}{2}\right)}, \\
Q_{p}\left[S_{\infty}^{c}\right] & =\frac{1}{\delta}\left(1+a \sqrt{2 \pi} e^{\frac{a^{2}}{2}} \Phi(a)\right), \\
Q_{p}\left[S_{\infty}^{l}\right] & =\frac{1}{c \delta^{*}} \sqrt{2 \pi} e^{\frac{\left(\Phi^{-1}(p)\right)^{2}}{2}}\left(\Phi\left(c-\Phi^{-1}(p)\right)-1+p\right),
\end{aligned}
$$

with $F_{X}$ the cdf of the Gamma distribution and

$$
\begin{aligned}
a & =\frac{\sigma \Phi^{-1}(p)}{\sqrt{2 \delta}}, \\
c & =\sigma \sqrt{\frac{2}{\delta^{*}}} .
\end{aligned}
$$

In Table 1 we show the quantiles of $S_{\infty}^{l}, S_{\infty}^{c}$ and $S_{\infty}$ in the case where $\delta=0.07$ and $\sigma=0.1$. These results can be compared with the results reported in Dhaene et al. (2002). The small differences we observe, can be explained as follows: Firstly, 


\begin{tabular}{|c|c|c|c|}
\hline$p$ & $Q_{p}\left[S_{\infty}^{l}\right]$ & $Q_{p}\left[S_{\infty}\right]$ & $Q_{p}\left[S_{\infty}^{c}\right]$ \\
\hline \hline 0.95 & 23.62 & 23.63 & 25.90 \\
0.975 & 26.09 & 26.13 & 29.34 \\
0.99 & 29.37 & 29.49 & 34.08 \\
0.995 & 31.90 & 32.10 & 37.86 \\
0.999 & 38.00 & 38.49 & 47.38 \\
\hline
\end{tabular}

Table 1: The table compares some selected exact quantiles of the constant perpetuity with the 'maximal variance' lower bound and upper bound approximations $(\delta=0.07$, $\sigma=0.1$ ).

\begin{tabular}{|c|c|c|c|}
\hline$p$ & $Q_{p}\left[S_{\infty}^{l}\right]$ & $Q_{p}\left[S_{\infty}\right]$ & $Q_{p}\left[S_{\infty}^{c}\right]$ \\
\hline \hline 0.25 & 11.13 & 11.07 & 9.34 \\
0.50 & 15.74 & 15.76 & 14.29 \\
0.75 & 23.51 & 23.50 & 23.11 \\
0.95 & 46.30 & 46.14 & 51.84 \\
0.99 & 79.64 & 80.71 & 100.45 \\
0.995 & 98.35 & 101.09 & 130.77 \\
\hline
\end{tabular}

Table 2: The table compares some selected exact quantiles of the constant perpetuity with the 'maximal variance' lower bound and upper bound approximations $(\delta=0.07$, $\sigma=0.2$ ).

these authors computed the quantiles of $S_{\infty}^{c}$ and $S_{\infty}^{l}$ by numerical evaluation of the expressions (24) and (26) with $t \rightarrow \infty$ and $\alpha(\tau)=1$. Secondly, they effectively used a slightly different conditioning variable, i.e. $\Lambda=\int_{0}^{\infty} e^{-\delta \tau} Z(\tau) d \tau$, whereas our explicit results rely on $\Lambda=\int_{0}^{\infty} e^{-\delta^{*} \tau} Z(\tau) d \tau$ as conditioning random variable.

In Table 2, we show quantiles of $S_{\infty}, S_{\infty}^{c}$ and $S_{\infty}^{l}$ but now for $\delta=0.07$ and $\sigma=0.2$. This example is interesting because it proves that for suitable choices of $\Lambda$, the cdf's of $S_{\infty}^{l}$ and $S_{\infty}$ do not necessarily cross only once. In this respect it is worthwhile to mention that Vanduffel et al. (2003) showed in a discrete setting that the cdf's of $S_{t}^{l}$ and $S_{t}^{c}$ can only cross in the region where their distribution functions take a value that is contained in the interval $\left[p^{-}, p^{+}\right]$for some $p^{-}$and $p^{+}>0$ leaving it as an open question whether this crossing point is unique or not. Exactly the same result can be drawn in the continuous setting, but despite the explicit expressions for the quantiles, we are still unable to answer satisfactorily the question concerning the uniqueness of the crossing point.

Vanduffel et al. (2005b) mentioned that in the discrete setting and using finite horizons, the 'maximal variance' comonotonic lower bound approximation provides an excellent fit for several risk measures associated with sums of lognormals. As visually seen from these tables, the same holds true in the continuous setting even when the time horizon goes to infinity. Hence our numerical comparisons provide some more support regarding the conclusions made in Vanduffel et al. (2005b).

\section{$5 \quad$ Asian options}

Until now we have considered closed-form comonotonic approximations for risk measures of continuous annuities. In this section we will show how these results can be 
used to derive closed-form expressions for upper and lower bounds for the price of a continuously sampled European-style arithmetic Asian option with fixed exercise prices.

We are not the first to investigate this problem. Vorst (1992) essentially used the fact that the geometric average is always smaller than the corresponding arithmetic average to derive closed-form expressions for upper and lower bounds. The upper bound has been considerably improved since then. We refer to Rogers \& Shi (1995), Nielsen \& Sandman (2003) and most recently to Lord (2006) where tight upper bounds have been provided. In this section we derive an upper bound in closedform but unfortunately this expression is only valid for a restricted choice for the parameters.

As far as the lower bound prices are concerned, using the technique of conditioning, these have been sharpened in a series of papers by Curran (1994), Rogers \& Shi (1995) and Thompson (1999). However all these lower bound approximations still involve the evaluation of a two-dimensional or one-dimensional integral. In this section we will derive another lower bound, which is also based on the technique of conditioning but does not require the evaluation of an integral anymore.

The risk neutral price of a European-style Asian option with fixed strike price $K$ and maturity $T$ with averaging starting at $t=0$ will be denoted by $A C(K, T)$ and is given by

$$
A C(K, T)=\frac{e^{-r T}}{T} E^{Q}\left[\left(\int_{0}^{T} A(\tau) d \tau-T \cdot K\right)_{+}\right],
$$

where $Q$ denotes the equivalent martingale measure, $r>0$ is the risk-free continuous rate and $A(\tau)$ is the price of the underlying risky asset at time $\tau$ for $\tau \in(0, T)$. We will also assume that $A(\tau)$ follows a geometric Brownian motion with drift $\mu-\frac{\sigma^{2}}{2}$ and volatility $\sigma$. It is well known that under the equivalent martingale measure $Q$ we find that $A(\tau)$ is given by

$$
A(\tau)=A(0) e^{Z(\tau)}
$$

where $Z(\tau)=\left(r-\frac{\sigma^{2}}{2}\right) \tau+\sigma B(\tau)$ is a Brownian motion with drift $r-\frac{\sigma^{2}}{2}$ and volatility $\sigma$.

We can also express $A C(K, T)$ in terms of a stop-loss premium of a continuous annuity as follows. From definition (7) for the continuous temporary annuity $S_{t}$ we find that

$$
\int_{0}^{T} A(\tau) d \tau=A(0) \int_{0}^{T} e^{Z(\tau)} d \tau=A(0) S_{T}
$$

but with $\delta$ given by

$$
\delta=-\left(r-\frac{\sigma^{2}}{2}\right)
$$

so that we can write

$$
A C(K, T)=\frac{e^{-r T}}{T} A(0) E^{Q}\left[\left(S_{T}-\frac{T \cdot K}{A(0)}\right)_{+}\right]
$$

Whilst an analytical expression for the call price (66) appears to be out of reach we can apply the bounds that were developed in Section 3.2 and Section 3.3. 
The upper bound price will be denoted by $A C^{c}(K, T)$ and can be simply found by applying (6) and (31) to (66) and using that in our risk neutral valuation context $\delta^{*}=-r$. Here, the probability level $p$ is determined by solving $Q_{p}\left[S_{T}^{c}\right]=\frac{T \cdot K}{A(0)}$ which can be done invoking equation (29). Unfortunately, it holds that $A C^{c}(K, T)$ only exists when $r<\frac{\sigma^{2}}{2}$ and this makes this upper bound of relatively little use.

We can also find a lower bound for $A C(K, T)$, obtained by conditioning on $\Lambda=$ $\sum_{i=1}^{n} \gamma\left(t_{i}\right) Z\left(t_{i}\right)$ as defined in Section 3.3.3. This lower bound price will be denoted by $A C^{l, s}(K, T)$, and a closed-form expression for it can be readily found by applying expression (6) and (48) to (66) where $\delta^{*}=-r$. In this case $p$ will follow from solving $Q_{p}\left[S_{T}^{l, s}\right]=\frac{T \cdot K}{A(0)}$ which can done using (44). Note that $A C^{l, s}(K, T)$ always exists and unlike the existing lower bound approaches that are based on conditioning does not require the evaluation of an integral.

In Table 3 we compare for an arithmetic Asian option our lower bound $A C^{l, s}(K, T)$ with the lower bound price from Rogers and Shi (1995) using Monte Carlo simulations as the benchmark. While Rogers \& Shi's analysis was valid for any choice for the conditioning rv $\Lambda$, in their numerical investigations they proposed $\Lambda=\int_{0}^{T} Z(\tau) d \tau$ as suitable choice to derive the lower bound prices. In contrast, we start by taking $\Lambda_{1}=\sum_{i=1}^{n} e^{r t_{i}} Z\left(t_{i}\right)$ with $t_{i}=\frac{t}{n}(i=1,2, \ldots, n)$ as choice for our conditioning rv. This choice is strongly inspired by the maximal variance approximation that we discussed in Section 2.2. The results for $A C^{l, s}(K, T)$ that are obtained using this choice for $\Lambda$ will for convenience be labelled as 'Maximal variance'. We also rely on the discussion in Section 2.2 to test the locally optimal $\Lambda_{2}=\sum_{i=1}^{n} e^{r t_{i}} \cdot \Phi^{\prime}\left(r_{i}^{1} \sigma \sqrt{t_{i}}-\Phi^{-1}(p)\right) Z_{t_{i}}$ as conditioning random variable where $r_{i}^{1}$ is the correlation between $\Lambda_{1}$ and $Z_{t_{i}}$. In this case we label the corresponding results for $A C^{l, s}(K, T)$ as 'Max CTE'.

Furthermore, we assume $\sigma=0.1$, an initial stock price of $A(0)=100$ and as expiry time we take $T=1$. We will also take $n=36$ which essentially means that we use the information of the underlying stock on a 10-daily basis when deriving $A C^{l, s}(K, T)$. The results show that the 'Maximal variance' and 'Maximal CTE' analytic bounds provide equally accurate results as in Rogers \& Shi (1995) and also that these are very close to the results obtained by Monte Carlo simulations. However the tied bound of Rogers \& Shiu (1995) or, equivalently, the one that was proposed in Thompson (1999) require the evaluation of a double or single integral, respectively. In contrast, our lower bound formula involves a sum with a fairly limited amount of terms only.

In Table 4 we repeat the exercise but now assuming $\sigma=0.3$. Also in this case we find a close correspondence between the results of Rogers \& Shi (1995) and our results. Our numerical experiments revealed that increasing $n$ or making other choices for the evaluation times $t_{i}$ did not improve the obtained bounds further.

In contrast, Table 5 where we take $\sigma=0.5$ provides some support for the observations made in Vanduffel et al. (2007) that for out-of the money options and in case the volatility is large, the 'Maximal CTE' lower bound approximations outperform the other approximations.

\section{Concluding remarks}

In Dhaene et al. (2002) it is shown that using comonotonic approximations for risk measures for constant continuous annuities in a Black\&Scholes market gives rise 


\begin{tabular}{|c|c|c|c|c|c|}
\hline \multicolumn{5}{|c|}{$\sigma=0.1$} \\
\hline$r$ & Strike & Maximal variance & Maximal CTE & Rogers and Shi & Monte Carlo \\
\hline \hline 0.05 & 90 & 11.951 & 11.951 & 11.951 & $11.953[0.0018]$ \\
\hline & 100 & 3.641 & 3.641 & 3.641 & $3.639[0.0013]$ \\
\hline & 110 & 0.331 & 0.331 & 0.331 & $0.331[0.0004]$ \\
\hline 0.09 & 90 & 13.385 & 13.385 & 13.385 & $13.388[0.0018]$ \\
\hline & 100 & 4.915 & 4.915 & 4.915 & $4.916[0.0015]$ \\
\hline & 110 & 0.630 & 0.630 & 0.630 & $0.629[0.0006]$ \\
\hline 0.15 & 90 & 15.399 & 15.399 & 15.399 & $15.402[0.0017]$ \\
\hline & 100 & 7.027 & 7.027 & 7.028 & $7.027[0.0016]$ \\
\hline & 110 & 1.413 & 1.413 & 1.413 & $1.412[0.0009]$ \\
\hline
\end{tabular}

Table 3: The table compares the prices of Continuous Asian Call Options using the 'maximal variance' and 'maximal CTE' lower bounds, results in Roger and Shi (1995) and the Monte Carlo simulations. The figures between brackets represent the standard error on the Monte Carlo result ( $\mathrm{T}=1$, averaging starts immediately).

\begin{tabular}{|c|c|c|c|c|c|}
\hline \multicolumn{5}{|c|}{$\sigma=0.3$} \\
\hline$r$ & Strike & Maximal variance & Maximal CTE & Rogers and Shi & Monte Carlo \\
\hline \hline 0.05 & 90 & 13.951 & 13.951 & 13.952 & $13.949[0.0047]$ \\
\hline & 100 & 7.943 & 7.943 & 7.944 & $7.937[0.0038]$ \\
\hline & 110 & 4.070 & 4.070 & 4.070 & $4.071[0.0028]$ \\
\hline 0.09 & 90 & 14.981 & 14.981 & 14.983 & $14.984[0.0047]$ \\
\hline & 100 & 8.826 & 8.826 & 8.827 & $8.823[0.0039]$ \\
\hline & 110 & 4.695 & 4.695 & 4.695 & $4.690[0.0030]$ \\
\hline 0.15 & 90 & 16.510 & 16.510 & 16.512 & $16.511[0.0048]$ \\
\hline & 100 & 10.208 & 10.208 & 10.208 & $10.207[0.0041]$ \\
\hline & 110 & 5.728 & 5.728 & 5.728 & $5.730[0.0032]$ \\
\hline
\end{tabular}

Table 4: The table compares the prices of Continuous Asian Call Options using the 'maximal variance' and 'maximal CTE' lower bounds, results in Roger and Shi (1995) and the Monte Carlo simulations. The figures between brackets represent the standard error on the Monte Carlo result. ( $\mathrm{T}=1$, averaging starts immediately).

\begin{tabular}{|c|c|c|c|c|c|}
\hline \multicolumn{5}{|c|}{$\sigma=0.5$} \\
\hline $\mathrm{r}$ & Strike & Maximal variance & Maximal CTE & Rogers and Shi & Monte Carlo \\
\hline 0.09 & 90 & 18.178 & 18.181 & 18.183 & $18.187[0.0076]$ \\
\hline & 100 & 13.019 & 13.020 & 13.022 & $13.027[0.0068]$ \\
\hline & 110 & 9.117 & 9.117 & 9.118 & $9.115[0.0059]$ \\
\hline & 150 & 1.927 & 1.930 & 1.924 & $1.931[0.0029]$ \\
\hline & 200 & 0.2566 & 0.2596 & 0.2548 & $0.2589[0.0011]$ \\
\hline
\end{tabular}

Table 5: The table compares the prices of Continuous Asian Call Options using the 'maximal variance' and 'maximal CTE' lower bounds, results in Rogers \& Shi (1995) and the Monte Carlo simulations. The figures between brackets represent the standard error on the Monte Carlo result ( $\mathrm{T}=1$, averaging starts immediately). 
to expressions that only involve the evaluation of a single integral. In this paper we showed that these integrals can sometimes be evaluated explicitly, hence yielding analytic approximations and bounds in this case. We compared these approximations with available explicit results for perpetuities.

We use our results to derive analytical formulas for upper and lower bound prices for continuously sampled Asian Options. The lower bound prices are at least as sharp as those from Rogers \& Shi (1995) but since they do not require any longer the evaluation of an integral they are extremely fast to obtain.

\section{Acknowledgments}

Jan Dhaene and Zhaoning Shang acknowledge the financial support by the Onderzoeksfonds K.U.Leuven (GOA/02: Actuariële, financiële en statistische aspecten van afhankelijkheden in verzekerings- en financiële portefeuilles). Steven Vanduffel and Jan Dhaene also acknowledge the financial support of Fortis (Fortis Chair in Financial and Actuarial Risk Management). The authors also want to thank an anonymous referee for helpful comments and in particual for a suggestion that appeared to be crucial in finding the lower bound approximation as proposed in Section 3.3.3.

\section{References}

[1] Curran, M. (1994) "Valuing Asian and portfolio options by conditioning on the geometric mean price", Management Science, 40(12), 1705-1711.

[2] Denuit, M., Dhaene, J. \& Van Wouwe, M. (1999). "The economics of insurance: a review and some recent developments", Mitteilungen der Schweiz. Aktuarvereinigung, 1999(2), 137-175.

[3] De Schepper, A.; Teunen, M.; Goovaerts, M.J. (1994). "An analytical inversion of a Laplace transform related to annuities certain", Insurance: Mathematics $\mathscr{E}$ Economics, 14(1), 33-37.

[4] De Schepper, A. (1995). "Stochastic Interest Rates and the Probabilistic Behaviour of Actuarial Functions", Doctoraal Proefschrift, K.U.L., 211 p.

[5] Dhaene, J., Denuit, M., Goovaerts, M.J., Kaas, R. \& Vyncke, D. (2002). "The concept of comonotonicity in actuarial science and finance: applications", Insurance: Mathematics \&3 Economics, 31(2), 133-161.

[6] Dhaene, J., Vanduffel, S., Tang, Q., Goovaerts, M.J., Kaas, R., Vyncke, D. (2006). "Risk measures and comonotonicity: a review", Stochastic Models, 22, 573-606.

[7] Dufresne, D. (1990). "The distribution of a perpetuity with applications to risk theory and pension funding", Scandinavian Actuarial Journal, 9, 39-79.

[8] Huang, H., Milevsky, M. \& Wang, J. (2004). "Ruined moments in your life: how good are the approximations?", Insurance: Mathematics \&6 Economics, 34(3), 421-447. 
[9] Kaas, R., Dhaene, J. \& Goovaerts, M. (2000). "Upper and lower bounds for sums of random variables", Insurance: Mathematics and Economics, 27, 151-168.

[10] Lord, R. (2006). "Partially exact and bounded approximations for arithmetic Asian options", Journal of Computational Finance, 10(2).

[11] Nielsen, J.A. \& Sandmann (2003). "Pricing bounds on Asian options", Journal of Financial and Quantitative Analysis, vol. 38, no. 2, pp. 449-473.

[12] Rogers, L.C.G. \& Shi, Z. (1995). "The value of an Asian option", Journal of Applied Probability, 32, 1077-1088

[13] Thompson, G. (1999). "Fast narrow bounds on the value of Asain options", Working paper, Centre for Financial Research, Judge Institute of Management Science, University of Cambridge.

[14] Vanduffel, S., Dhaene, J., Goovaerts, M. (2005a). "On the evaluation of Saving Consumption Plans", Journal of Pension Economics and Finance, 4(1), 17-30.

[15] Vanduffel, S., Hoedemakers, T. \& Dhaene, J. (2005b). "Comparing approximations for sums of non-independent lognormal random variables", North American Actuarial Journal, 9(4), 71-82.

[16] Vanduffel, S., Chen, X. Dhaene, J., Goovaerts, M.J., Kaas, R., Valdez, E. (2006). Optimal Approximations for Risk Measures of Sums of Lognormals based on Conditional Expectations, Journal of Computational and Applied Mathematics, in press.

[17] Vanduffel, S., Dhaene, J., Goovaerts, M. \& Kaas, R. (2002). "The hurdle-race problem", Insurance: Mathematics and Economics, 33(2), 405-413.

[18] Vorst, T. (1992). "Prices and Hedge Ratios of Average Exchange Rate Options", International Review of Financial Analysis, vol. 1, no. 3, pp. 179-193.

\section{Appendix A}

In this appendix, we prove Equation (29). From equation (24), it follows that:

$$
Q_{p}\left[S_{t}^{c}\right]=\int_{0}^{t} e^{-\delta \tau+\sigma \sqrt{\tau} \Phi^{-1}(p)} d \tau
$$

By substituting $y=\sqrt{\tau}$ we find that:

$$
\begin{aligned}
Q_{p}\left[S_{t}^{c}\right] & =\int_{0}^{\sqrt{t}} 2 y e^{-\delta y^{2}+\sigma \Phi^{-1}(p) y} d y \\
& =2 \int_{0}^{\sqrt{t}} y e^{-\frac{\left(y-\frac{\sigma \Phi^{-1}(p)}{2 \delta}\right)^{2}}{1 / \delta}+\frac{\sigma^{2}\left(\Phi^{-1}(p)\right)^{2}}{4 \delta}} d y .
\end{aligned}
$$


Now let $z=\left(y-\frac{\sigma \Phi^{-1}(p)}{2 \delta}\right) /(1 / \sqrt{2 \delta})$ and $a=\frac{\sigma \Phi^{-1}(p)}{\sqrt{2 \delta}}$. Then, we obtain that:

$$
\begin{aligned}
Q_{p}\left[S_{t}^{c}\right] & =2 e^{a^{2} / 2} \int_{-a}^{\sqrt{2 \delta t}-a}\left(\frac{1}{\sqrt{2 \delta}} z+\frac{\sigma \Phi^{-1}(p)}{2 \delta}\right) e^{-z^{2} / 2} \frac{1}{\sqrt{2 \delta}} d z \\
& =e^{a^{2} / 2} \int_{-a}^{\sqrt{2 \delta t}-a}\left(\frac{1}{\delta} z+\frac{1}{\delta} a\right) e^{-z^{2} / 2} d z \\
& =\frac{1}{\delta} e^{a^{2} / 2}\left[\int_{-a}^{\sqrt{2 \delta t}-a}-d e^{-z^{2} / 2}+\int_{-a}^{\sqrt{2 \delta t}-a} a e^{-z^{2} / 2} d z\right], \\
& =\frac{1}{\delta} e^{a^{2} / 2}\left[\left(-\left.e^{-z^{2} / 2}\right|_{-a} ^{\sqrt{2 \delta t}-a}\right)+a \sqrt{2 \pi} \int_{-a}^{\sqrt{2 \delta t}-a} \frac{1}{\sqrt{2 \pi}} e^{-z^{2} / 2} d z\right] \\
& =\frac{1}{\delta}-\frac{1}{\delta} e^{-\delta t+\sigma \sqrt{t} \Phi^{-1}(p)}+\frac{1}{\delta} \sqrt{2 \pi} a e^{a^{2} / 2}(\Phi[\sqrt{2 \delta t}-a]-\Phi[-a])
\end{aligned}
$$

with $\Phi$ the cdf of a standard normal random variable and this completes the proof. 